\title{
Effective Scalable and Integrative Geocoding for Massive Address Datasets
}

\author{
Sina Rashidian \\ Stony Brook University \\ sina.rashidian@stonybrook.edu
}

\author{
Xinyu Dong \\ Stony Brook University \\ xinyu.dong.1@stonybrook.edu
}

\author{
Amogh Avadhani \\ Stony Brook University \\ amogh.avadhani@stonybrook.edu
}

\author{
Prachi Poddar \\ Stony Brook University \\ prachi.poddar@stonybrook.edu
}

\author{
Fusheng Wang \\ Stony Brook University \\ fusheng.wang@stonybrook.edu
}

\begin{abstract}
With increased accessibility of large scale open data, public health studies are able to take advantage of integrative spatial big data to increase the spatial resolution to community or neighborhood level. One critical information for such studies is the large number of addresses of patients, which is private and highly sensitive. Geocoding such massive private addresses poses major challenges for public health researchers. Many geocoders provide only Web APIs which require sending private addresses over the Internet, which is not feasible. Commercial geocoders require high licensing fee and often have limitations on daily usage, which becomes a major hurdle for researchers. Scalability is another major challenge for large scale address dataset. In this paper, we present EaserGeocoder, a novel open source geocoder for effectively geocoding massive address datasets. EaserGeocoder takes an integrative approach by using multiple references based on open address data sources contributed by governments or communities. It takes a machine learning approach to automatically find the best answer from candidates produced by multiple references. The system provides high scalability through parallel processing. Our comparative studies demonstrate EaserGeocoder outperforms open source geocoders and is comparable to commercial ones in terms of both accuracy and error. It provides a cost-effective and feasible solution for large scale public health studies.
\end{abstract}

\section{CCS CONCEPTS}

\section{- Information systems $\rightarrow$ Geographic information systems;}

\section{KEYWORDS}

Geographic Information System, Geocoding, Text Searching

\section{ACM Reference format:}

Sina Rashidian, Xinyu Dong, Amogh Avadhani, Prachi Poddar, and Fusheng Wang. 2017. Effective Scalable and Integrative Geocoding for Massive Address Datasets. In Proceedings of SIGSPATIAL'17, Los Angeles Area, CA, USA, November 7-10, 2017, 10 pages.

DOI: $10.1145 / 3139958.3139986$

Permission to make digital or hard copies of all or part of this work for personal or classroom use is granted without fee provided that copies are not made or distributed for profit or commercial advantage and that copies bear this notice and the full citation on the first page. Copyrights for components of this work owned by others than ACM must be honored. Abstracting with credit is permitted. To copy otherwise, or republish, to post on servers or to redistribute to lists, requires prior specific permission and/or a fee. Request permissions from permissions@acm.org.

SIGSPATIAL'17, Los Angeles Area, CA, USA

(C) 2017 ACM. 978-1-4503-5490-5/17/11 ..\$15.00

DOI: $10.1145 / 3139958.3139986$

\section{INTRODUCTION}

Spatial big data including health data are now widely accessible with government open data initiatives, geo-crowdsourcing, and social media, with high spatial resolution even at street level. Traditional public health studies, however, often use low spatial resolution (e.g., county/zip code) due to the lack of high resolution data and lack of efficient and scalable methods. Consolidating diverse large scale spatial data sources will significantly enhance public health studies at community or neighborhood level [20,21].

One example patient data source is New York State patient data collected by New York State data from Statewide Planning and Research Cooperative System (SPARCS) [3], which include patient level detail on patient characteristics, diagnoses and treatments, services, and charges for each hospital inpatient stay and outpatient, together with street address of each patient. The data collection started 35 years ago, and it is an ongoing effort. The records collected each year are more than 20 million.

The immediate goal for integrative spatial big data based public health studies is to geocode addresses of patients to have location information for each patient to support high resolution spatial data analytics. This is faced by major challenges.

The first major challenge is patients' privacy. The address of a patient is part of the patient's Protected Health Information (PHI) data $[9,34]$, which must be managed and protected in a Health Insurance Portability and Accountability Act (HIPPA) compliance environment. Sharing patients' addresses over the Internet is prohibited unless the Internet communication is approved for compliance. This makes it impossible to use Web API based geocoding tools as they require sending data over the Internet.

The second major challenge is the high cost for commercial geocoding. Geocoding with Google API [7] is volume based, which could cost more than 100K USD for geocoding 10 years' SPARCS data. This provides a major hurdle for academic researchers.

The third major challenge is efficiency. Based on our study, common geocoders have limited scalability for massive number of addresses, and open source ones lack the capability of parallel processing. As a result, it could take weeks or longer for large scale addresses.

Finally, patients' addresses are manually input data which come with noise and typos. Effective techniques to handle such noisy addresses are critical to achieve high accuracy for geocoding.

As a result, existing commercial geocoding providers either could not handle the privacy or come with high licensing fee, and open source ones are not viable for scalability or accuracy. There is a 
SIGSPATIAL'17, November 7-10, 2017, Los Angeles Area, CA, USA major demand for a new open scalable geocoding tool that can support geocoding massive private addresses with high accuracy and low cost.

Increased availability of big spatial data provides multiple open reference address datasets, including open government data such as The TIGER/Line Shapefiles [15] from US Census Bureau, county owned tax parcel data [22], State specific data [11], community generated data such as OpenStreetMap [14] and Open Address [12], among others. All the data sources are open and free, and being updated on a continuous basis.

While each dataset is produced for different purpose and may not be sufficient to support geocoding, we believe that the datasets could complement each other and achieve significant accuracy using an integrative approach.

This motivates us to develop an effective and scalable integrative geocoder for massive address datasets (EaserGeocoder). The EaserGeocoder takes advantage of multiple open data sources, including TIGER, OpenStreetMap, OpenAddresses and NYS Street Address Mapping (SAM). We take an integrative approach by searching each dataset for the closest match, to generate a candidate list. We propose a novel machine learning based approach to automatically rank candidates to generate the final answer by learning from a public address data with a comprehensive set of address features. Our contributions are summarized as follows.

- We develop an integrative geocoding model capable of using multiple reference sources complement each other to achieve high accuracy and minimize error.

- We propose a novel machine learning based method for solving the problem of choosing the best answer by learning from a ground truth dataset to produce a prediction model based on Gradient Tree Boosting.

- We take advantage of parallel processing and in-memory caching to achieve high scalability.

- The system is open source and can be conveniently deployed within a HIPPA compliant environment.

\section{RELATED WORK}

The geocoding process has been studied in $[28,31]$, and work in [23] discussed associated errors. Influence of reference sources is discussed in [41], and influence of environment and external factors is discussed in [18]. Work in [29,30] proposes strategies to improve geocoding process. How to evaluate geocoding systems has been discussed in [27, 38, 39, 42]. Recently, design of application specific geocoding system has attracted much interest [17, 19, 33].

Geocoding is considered as an initial process to generate input data to support other studies such as public health research [20,21, $36,37]$. Different methods for linking and binding geocoding data to records are discussed in [35] and impact of geocoding error and its propagation through final results is analyzed in [40].

There are many commercial geocoding systems available such as $[4,7,8,10,24]$. Open source goecoding systems include $[5,6,13]$. We will compare our approach to these systems in our work.

\section{OVERVIEW}

We first present a summary of major elements in classic geocoding model, and common geocoding methods and references. Then we
S. Rashidian et al.

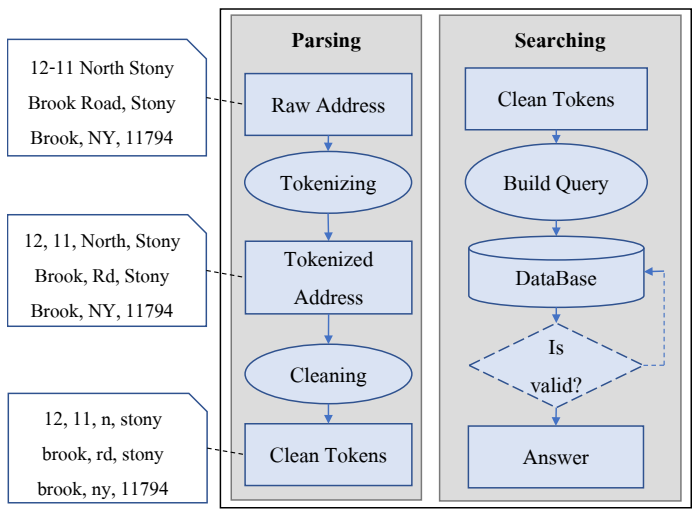

Figure 1: Classic Geocoding Model

introduce our Integrative Geocoding Model which is a combination of geocoding methods with innovative ideas for solving classic model flaws.

\subsection{Classic Geocoding Model}

Typical Geocoding Model Generally, a geocoding process has two major steps: parsing and searching. Parsing consists of tokenizing, cleaning, and standardization; searching includes sending query and analyzing the response. Parsing section breaks input address into small tokens or elements. As shown in Figure 1, for an input address, the parser will break it into address elements such as prefix, postfix, city name, county name, zip code and etc. In most geocoding studies, address features, elements, components, and tokens are used interchangeably [4, 41]. After the input address is parsed, clean tokens are sent to a database for finding similar matches. Normally, these models assign sum of similar features weights as the score to each match. Features weights are pre-defined and manually customizable by final user [39]. The score will show whether a match is acceptable or not.

Traditional Geocoding Methods Geocoding methods rely on underlying reference data. There are commonly three types of reference data: linear-based, polygon-based and point-based [28]. In linear-based format, each line is represented as a set of geolocation points. For extracting geolocation of one specific point, interpolation methods are needed to estimate the location of the given point. In this case, accuracy depends on precision of interpolation methods and density of points per unit length in the reference dataset. Linear interpolation is the most common interpolation method to apply on these reference datasets. Using street network maps along with linear interpolation is known as traditional geocoding which is widely used in geocoding systems [18, 36, 42].

In polygon-based reference data, boundary points of shapes are given. Sometimes, the term parcel is used with the same meaning as having a polygon-based reference data. The polygon in this case could belong to a building, village, city which could lead to different accuracy. These sources could be very useful for visualization projects as they have shape-related information. In geocoding systems, normally the centroid of the polygon/parcel is chosen as its geolocation for that address. However, this conversion is not error 


\section{Effective Scalable and Integrative Geocoding}

prone and could lead to some errors in results, since the residence location is not always in the centroid of the parcel [32, 35, 41].

For point-based reference data sources, the exact location of an address is assumed to be available. These references have high accuracy for exact-matched addresses and are easy to use since they are only returning one geolocation for the address. These sources along with centroid locations of polygon-based references are considred as exact-match references in this study.

\subsection{Geocoding Metrics}

Match rate in geocoding process, refers to the rate of acceptable results returned by a geocoder. Although, this term used in many literatures, it tends to be vague due to lack of precise definition of acceptable result $[41,42]$. Generally, geocoders assign scores to each result in a scale of 100 , and based on the specified matching threshold, the result could be considered as acceptable or not. Score is the sum of predefined weights for equal features between an input query and result [39]. Such definition does not provide sufficient information on how close the results are to the "ground truth" points. Another important concept in geocoding is Spatial accuracy, positional accuracy or positional error which is used in literatures with the same meaning of average distance between results from geocoding process and ground truth geolocation [31, 32, 35, 36, 39, 41, 42]. Although, match rate and positional accuracy have been studied in many literatures, fewer of them report exact numbers for positional accuracy and there was less attention to this measurement till recently [32]. Some studies only considered scores generated by geocoding tools as their baseline and metric for comparisons. In particular, if the street name and one higher level geographical element (such as zip code or city name) are the same, it will generate a high score and could be considered as correct based on the threshold for acceptable results defined by users. However, there could be cases where match score is high, but the geolocation returned by geocoding tool is not close enough. Reasons root in vague and imprecise definition of scoring system and lack of accuracy in underlying references $[32,35]$. In our study, instead of using "match rate", we provide a clearer definition of accuracy in public health studies by combining match rate and positional accuracy.

\subsection{Integrative Geocoding Model}

We propose an integrative geocoding model, which utilizes multiple open data sources as underlying references, including linear-based, polygon-based and point-based, contributed by either governments or communities. An immediate benefit is that there is no cost for data which makes it possible to develop an open source geocoding system. Although one reference may not be as complete as commercial ones, multiple references could complement each other and have the potential to support geocoding with high accuracy.

Our goal is to extract knowledge as much as possible from each reference by applying corresponding geocoding method. As these data sources are highly different, and each data source has its own features and characteristics, merging them together is not likely or could lead to losing information. Instead, for each reference, we take the most optimized approach based on its characteristics to generate a candidate independently, leaving the data source unchanged except pre-data cleaning and standardization. A candidate
SIGSPATIAL'17, November 7-10, 2017, Los Angeles Area, CA, USA

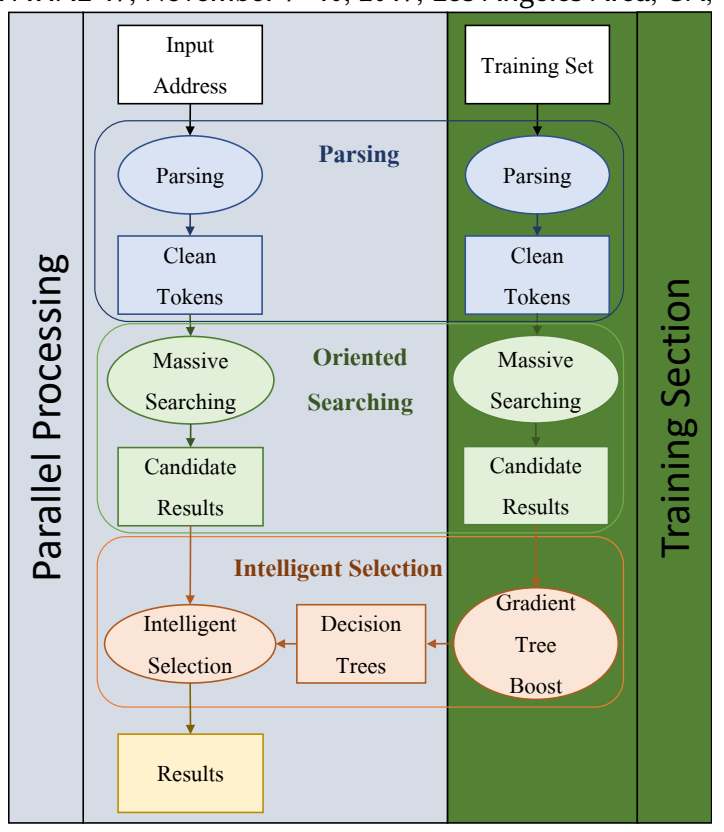

Figure 2: Integrative Geocoding Model

is the most similar match from a source. Then we decide between different candidates which one is the best to choose as the final result. As in Figure 2, the searching consists of two modules, Oriented Searching for each individual reference and Intelligent Selection for selecting the final answer.

Oriented Searching. EaserGeocoder extracts most knowledge from each individual data source. First, we prioritize the order for searching reference datasets, based on their increasing spatial error. We measured spatial error of each reference separately. Secondly, we send optimized customized query to each exact-match reference data, looking for a perfect answer. The reasons behind generating separate queries are discussed in Section 5.2. Sequence of searching in data sources is important, since if we find a perfect match in any exact-match source, the searching is stopped, and we return that response. Perfect match definition is dependent on the characteristics of the data source and the input query. For instance, in Tax Parcels, since many zip code values are missing, its equality is not required. Thirdly, if there is no perfect match from any of the exact-match references, we gather most similar matches from each source and generate a candidate list as input for Intelligent Answer Selection.

Intelligent Answer Selection. With a list of candidates from Oriented Search, the next step is to identify the best one as the final answer. This task is challenging due to the potential incompleteness or noise of data sources along with their different characteristics.

As mentioned earlier, a common traditional approach for answer selection is based on assigning scores by pre-defined weights for data sources with equal or similar address elements between input query and each candidate [30,39]. In our approach, different reference sources do not share same set of address elements, and pre-defined weighting based approach is not optimized in this state. For example, one reference source has city name, but in the other one it is partially or entirely missing. Instead, we propose to use 
SIGSPATIAL'17, November 7-10, 2017, Los Angeles Area, CA, USA

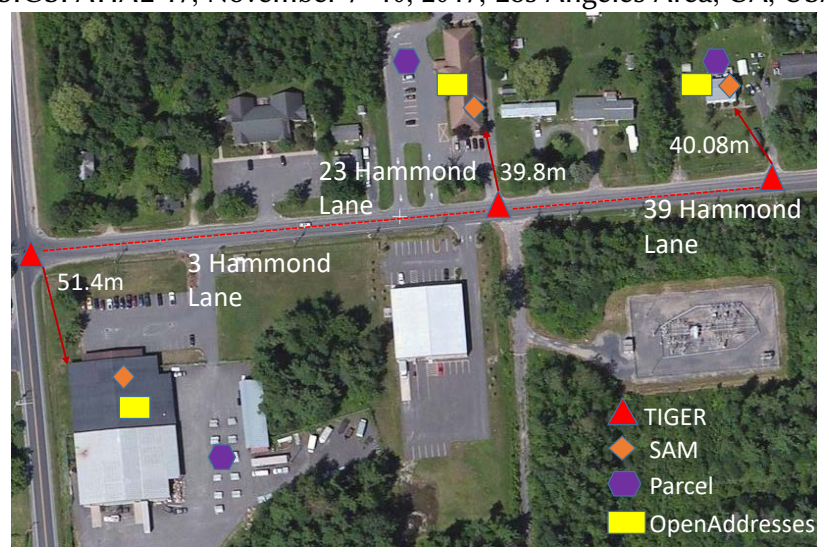

Figure 3: Sample visualization of results from different data sources

a machine learning based approach to learn from a training set of candidates in order to find the best one. In this way, data sources characteristics are considered in the selection process, so it becomes more intelligent and we could extract more data from them. This idea and the implementation details are elaborated in Section 5.4.

As a summary, our model looks for using multiple reference data sources with regards to their flaws. We study their characteristics, to learn their weaknesses and strength for optimizing our search methods. In Section 4, we introduce data references used in our system along with their features and specifications. In Section 5, we discuss our searching strategies with details for maximizing the accuracy. Finally, in Section 6, we show performance and functionality of this new model by comparing it with high tech commercial vendors and open source geocoding systems.

\section{OPEN REFERENCE DATA}

Open data initiatives and crowdsourcing have produced highly valuable geospatial data, including open government data, tax parcel data, community contributed data, and state specific data. We will focus on following data sources, but our approach can be generalized to incorporate more data sources if available. Note that we only support geocoding for US addresses at the moment. We will discuss merits and disadvantages of each one with more details.

\subsection{TIGER}

Topologically Integrated Geographic Encoding and Referencing (TIGER)[15] provides a US national data source (TIGER/Line Shapefiles) which consists of spatial extracts from the Census Bureau's MAF/TIGER database. The shapefiles include polygon boundaries of geographic areas and features and linear features including roads and hydrography.

It provides a street network map which contain a set of geolocations for each street, along with features like zip code and address range for each side. This street network map widely used in geocoding systems, since it has a vast coverage and data is reliable [36]. We use this street network map as one of our references.

TIGER suffers from drawbacks of linear-based references discussing in Section 4.5. Furthermore, it is based on county and zip
S. Rashidian et al.

code and does not provide any information about city names. If the input query has city name and does not have zip code or county information, it would be difficult to find its geolocation.

\subsection{Tax Parcels}

A tax parcel [22] is a legal description and division of land with the main purpose of providing a precise unit of taxation in support of taxpayers. It is also commonly referred to as real property or real estate map. The complete tax parcels varies between counties, and some counties may not make the data available. In this paper, we use NY State parcel as a driving example.

We will use Tax Parcels data as a point-based reference. It consists of exact matching addresses with one point representing for each address. Major drawbacks of this data source are missing many buildings addresses and partial information for many records such as lack of zip code[32, 41, 42].

\subsection{Community Contributed Datasets}

We consider two rich datasets contributed from communities including Open Street Map (OSM) [14] from geocrowdsourcing, and OpenAddresses [12] by individuals and organizations.

OpenStreetMap(OSM) is a large-scale world map project through extensive collaborative contribution from a large number of community users. It contains spatial representation of geometric features such as lakes, forests, buildings and roads. Spatial objects are represented by a specific type such as points, lines and polygons, therefore it has point-based, polygon-based and linear-based reference inside of it. It has more than 2.7 billion objects (nodes, ways and relations) with 2 million registered users.

OpenAddresses(OA) is a collection of authoritative data for address locations around the world, and is open to public contributions. It contains more than 466 million addresses worldwide. For each input address it has one point as its geolocation.

These community based datasets could complement government published data, but could come with much noise, formatting issues and inconsistency. For example, an OSM may contain wrong markups. OA aggregates many data sources input by users, which could lead to formatting problems. Thus, cleaning is a critical step for using such data sources.

\subsection{Local State Specific Dataset: Street and Address Maintenance(SAM) Program (NY)}

Besides, each state may contribute its own local datasets for special purposes. For example, NY Street and Address Maintenance Program (SAM)[11] collects street and address point data of New York State for Next-Generation 911 emergency systems.

Such data are often limited in coverage, but have their own unique features. For example, SAM only provides public data for 51 counties of 62 counties in NY State. While such data themselves cannot be used as a single data source for geocoding, they may be used to enhance geocoding with other references. We will take this dataset as an example of local government data sources. This data source contains both address points and street lines. We only used address points for the purpose of this study. 
Effective Scalable and Integrative Geocoding

\subsection{Spatial Error in References}

Imprecise geolocations and spatial error could be in any type of references for a variety of reasons, such as being outdated or mistakes in gathering data. However, among references types, linear-based ones tend to have higher spatial error [16, 23, 28, 32]. First, interpolation algorithms used in them produce error, as it assumes all buildings have same length which is not correct (parcel homogeneity). Secondly, these references contain address ranges for each street, however, these address ranges are an upper bound and all buildings in an address range do not necessarily exist. This could lead to wrong estimation of number of buildings and impair the accuracy. Thirdly, it is common to specify offsets for start and end of a street to estimate building locations more accurately, as there are empty space between the intersection and first building in the street. Unfortunately, there is no standard for these offsets amounts which is a problem itself. On the other hand, in exact-match address sources, spatial error reason only roots in using centroid of parcels as their geolocation.

\subsection{Reference Data Normalization}

Each reference source has its own set of data elements, format and style of data representation. The reference data have to normalized before before being importing to the database of the search engine, normally with these steps: 1) extracting geolocation in latitude and longitude format, 2) reorganizing their set of elements, 3) converting all postfix, predirectional, suffix, and postdirectional tokens to their abbreviated format, 4) making capitalization of strings, and 5) removing unnecessary tokens. By making data consistent and removing noises, we minimize the risk of misleading search in these valuable sources. For example, in some cases, data are not practically beneficial as they have too much missing information or contain incorrect labels.

Overlaps can happen between data sources. For instance, OSM data source imports TIGER data, and OpenAddresses uses partial SAM data. After performing analysis on these sources, we observed that OSM data source contribution is negligible in integrative process. In other words, the set of TIGER, OA, Tax Parcels, and SAM sources could cover all addresses contained in OSM.

As shown in an example of data representation in these data sources in Figure 3, linear-based references such as TIGER generally have higher spatial error than exact match references like SAM, Tax Parcels and OA.

In a summary, each data source has some virtues and drawbacks and could not be used independently as the sole source; instead, an integrative approach could build a rich foundation of data references (and free of charge) by taking advantage of the benefits and avoiding the drawbacks.

\subsection{Search Engine}

The major task for address matching is text searching and information extraction from reference data sources. To perform a fast and customized text searching, we need a data management system for effective text search. We employ Apache Solr [1] as our database implementation, as it is a powerful text oriented search engine with text indexing for efficient text search. Although the overhead for index building is high, building indexes is a one-time process and
SIGSPATIAL'17, November 7-10, 2017, Los Angeles Area, CA, USA the data update is infrequent. Solr also provides caching capabilities which significantly reduces I/O cost. We experimented relational database based approach, which has limited text search capabilities and can generate lots of SQL queries degrading the performance.

For each data source we create a separate core in Solr, maintaining a separate set of features for each reference data.

\section{INTEGRATIVE GEOCODING}

Our integrative geocoding has following steps. 1) Parsing: We parse the input address to a set of features by tokenizing, cleaning and standardization (5.1); 2) Customized Query: A customized query will be generated for each individual reference data source; 3) Database search: The query is submitted to the database to search across all data sources (5.2). If a perfect match is found, the result will be returned as the final answer; 4) Relaxed Search: If an exact search does not return an answer, a query relaxation will be performed by relaxing the search conditions such as relaxing the range. The relaxed search will return a list of best results (or candidates) from different sources (5.3). 5) Intelligent Answer Selection. To choose the answer from the candidates, we take a machine learning approach by pre-training a public address datasets with Gradient Tree Boost algorithm to build the classifier. Based on the classifier, we classify the candidates to specify the answer (5.4). Besides, EaserGeocoder makes the process highly scalable through parallelized processing (5.5).

\subsection{Input Address Parsing}

Parsing consists of tokenizing, cleaning and standardization of an input address and breaking it into several elements. In summary, we apply same normalization and standardization techniques discussed in Section 4.6 on input queries. Normalized features and format in input queries and reference data will help on effective searching and comparison. For instance, consider the example of parsing query " 1300 North Star Rd" discussed in [41]. Here "North Star" is a combined street name. If a parser detects "North" as a prefix of street name "Star", it would impair the search quality. Our approach will reformat both sides (input query and reference data) thus such a problem never occurs. The usual approach for solving this problem is parsing input address in multiple set of elements, searching each set separately and choosing the one with highest score as the correct set [4]. Our approach is more efficient, since it only parses input in one set of elements and does not require search for multiple sets.

Another example is two-part building number (e.g., "71-20"), which is very common in general format of addressing. The two parts are usually separated by a symbol like hyphen sign. We call the first token the base number, second token as the street number and both of them in their original format building number. A common mistake in input addresses is both parts are merged into one token without any separators, which misleads geocoding systems (e.g, "7120"). Although this problem could be widespread in some dataset inputs, fewer literatures discuss it in details. We propose to identify specialized address patterns of neighborhoods to solve this problem.

Identification of Neighborhoods with Specialized Address Patterns. Here we use dash combined address numbers as an example, but this can be generalized. We first identify neighborhoods where more than $95 \%$ of their addresses consist of two-part building 
SIGSPATIAL'17, November 7-10, 2017, Los Angeles Area, CA, USA number. For this purpose, we go through three combinations of neighborhoods: 1) county, zip code and street name, 2) zip code and street name; and 3) only zip code. We perform a preprocessing on all of these combination areas to extract their building number patterns if applicable. This will identify a collection of neighborhoods where such patterns are high enough. Based on this, the number format of input address in such neighborhoods will be corrected. For instance, all addresses in zip code 11351 have two-part building number formatting. As a result, an input address with this zip code and just one part building number is invalid and would be modified. Similar examples are for the "Caldwell Ave with zip code 11378 " or in "Queens county, 138th rd with zip code 11413". The idea of finding patterns in advance, which described here, could be expanded further to cover all neighborhoods.

\subsection{Customized Query Generation}

Based on characteristics of each data source, we generate specific query for that source. For instance, in TIGER data, city name is not available, thus it will not be used in the query. For Tax Parcels, there are many missing zip codes, adding zip code for such data source as mandatory filter could be misleading and impair searching quality. EaserGeocoder generates customized queries based on features of each reference to increase both accuracy and performance.

In some reference sources, a single customized query could not retrieve all needed information for a reference. EaserGeocoder will consider multiple scenarios and generate a set of customized queries to overcome this problem.

\subsection{Query Relaxation}

Searching is prioritized based on finding exact-match results.Our data sources consist of multiple exact-match sources and one street network source. First, we search through each exact-match data sources, to see if there is a perfect match or not. The order of searching in data sources is specified by their positional accuracy as shown in Table 4, in an ascending order. If we could not find a perfect match among exact-match sources, we identify nearest results among all references.

When the exact building could not be found, we look for the nearest ones. It is common that in exact-match data sources, some buildings in a street could be missing [41]. In this case, we search to see whether we could find any other building on the same street. For this purpose, we expand the scope of our search iteratively if we could not locate a near building. Furthermore, we prefer to find a building on same side of the street, so we apply some penalty on buildings on the other side of the street in the searching process based on their parities (even/odd). The rationale behind this penalty is that address ranges on both sides are not necessarily synchronized with each other. For instance, when there is no data available for building number 10 on one street, geolocation of building number 14 is preferred to buildings 11 and 13. In this case, we are unable to locate the exact location of the building. However, the building 14 could be close enough and its geolocation can be used as an approximation. The spatial proximity can be further verified by a classifier (discussed in next sections).

In a summary, the following approaches are taken to overcome conditions and limitations of open source datasets to maximize

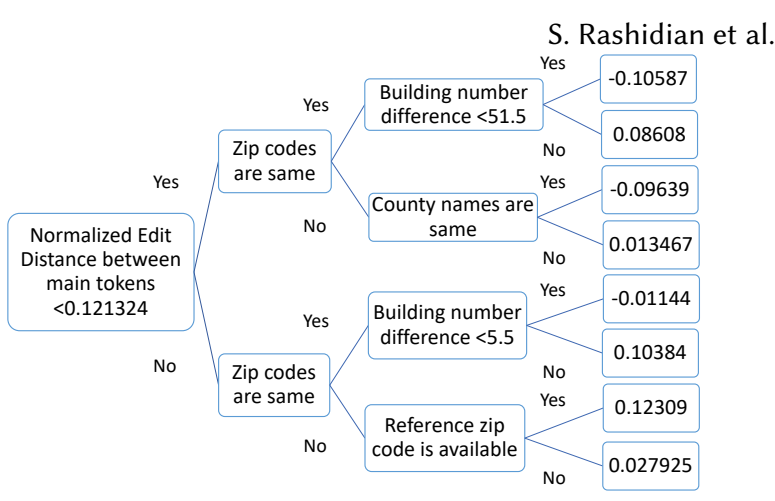

Figure 4: A Sample Decision Tree

search effectiveness. First, for each data source, customized query is built based on the characteristics of individual data source. Secondly, for features partially available in a data source, we put them as mandatory, optional or not required based on that data source and its features. Finally, for exact-match data sources, we start by a small search scope, and iteratively expand the scope until a close match is found. At the end, we either find a perfect match, or we have a couple of candidates needed to be compared to find the answer to the input address.

\subsection{Intelligent Answer Selection}

The algorithm of choosing result among candidates, directly impacts the accuracy of the result. So far for proposing candidates, only similarity between input query and existing data in each reference data source is considered. To improve the accuracy of result identification, we need to consider more factors beyond similarity. Consider an example input address " 21 Airport Rd, Binghamton, NY 13901”. This address finds a perfect match in TIGER, and a partial match with OA reference, which does not contain zip code. The matched record from TIGER has a spatial error around $450 \mathrm{~m}$ and the matched record from OA has a spatial error around $350 \mathrm{~m}$ based on the true location. Consider another example input address " 510 Main St, Oneida, NY 13421". Similarly, there is a perfect match in TIGER, and a partial match in OA where again only zip code is unavailable. Spatial error from TIGER is only $50 \mathrm{~m}$, but the spatial error from $\mathrm{OA}$ is more than $30 \mathrm{~km}$, as the returned result belongs to another zip code(13501). The traditional approach uses a set of fixed rules and pre-defined weights for calculating score for each response. It only focuses on similarity of address elements between input address and matching address in data sources. Thus, if two input addresses have same structures, the same reference data source will be chosen for returning the answer. If TIGER is chosen, the second address will have high accuracy, but the first one will not have the optimal result. If $\mathrm{OA}$ is chosen, the first address will return the optimal result, but the second address will return a result with significant error. However, it is possible to choose optimal results in both cases by having an intelligent answer selection.

There are many factors to be considered for choosing the best answer, and a manual customization approach will be highly difficult. For instance, as discussed in [18], population density of areas has impact on geocoding process. Addresses from one zip code could have reasonable geocoding accuracy, but those from another one 


\section{Effective Scalable and Integrative Geocoding}

may not. To provide an automated approach for choosing answers from candidates, we propose a machine learning based approach by learning from a ground truth dataset to produce a prediction model based on Gradient Tree Boosting, and use the model to classify candidates to generate best answer. The model will consider features including building number; street name; base number, prefix, postfix; city name, county name, and zip code. One essential benefit for learning such a model is considering spatial proximity factors for improving the choice of the best candidate.

"Ground Truth"

Generally, for datasets without geolocations for addresses, the problem of actual ground truth rises. It is impossible to collect the geoloations manually using positioning sensors such as GPS due to the overwhelming scale. In this model, we take an approximated approach by using results from Google Geocoding as the "ground truth". This could lead to immediate problems. If Google could not geocode an address, it modifies it to find the best result and return the answer based on the modified query. We replace the original address with the modified address. If Google Geocoder could not geocode one address at all, the address must be removed from the dataset, as the address itself is likely to have problem. By replacing or removing addresses, we will avoid problematic addresses and create a training dataset to support intelligent result selection.

\section{Training Dataset}

We collected 19,095 random samples of physician addresses in New York State provided by The Centers for Medicare and Medicaid Services (CMS)[2]. The "ground truth" of geolocations for this dataset is generated from Google geocoding as described above. Given this dataset to our system, we generate four geocoding candidates for each address using four references and calculate the distance between each candidate and the "ground truth" geolocations. We take 400 meters as an error threshold to classify each candidate as "correct" or "wrong". Then we defined a feature vector estimated by comparing input address and matching record for each candidate, to form the features for the classifier.

\section{Features}

For each input address, each reference will return a candidate and the corresponding record in the reference data source that produces the result. For example, for address " 510 Main St, Oneida, NY 13421", the matching record from TIGER is "500-520 Main St, NY 13421", and the distance between the candidate and the ground truth is $450 \mathrm{~m}$, thus the correctness label is "wrong". We compare the matching record with the original address from the ground truth dataset, and generate a set of features used for the classifier based on distance functions defined for each address element.

Absolute Distance between Building Numbers. As the nearest building on the same side of the street to input building is preferable, we calculate the absolute distance between building numbers as their distance. If a street number is on the other side, it is subject to some penalty as mentioned earlier.

Normalized Levenshtein Distance for Street, City and County Names. There are many cases that the string saved in data sources could be very similar to the input, but not an exact match. For instance, the street name of an input address "Saint Lawrence" may have variations such as "St.Lawrence", "Saint Lawrence", or "St Lawrence"
SIGSPATIAL'17, November 7-10, 2017, Los Angeles Area, CA, USA which will be compared to the matching record from the reference source. We use Normalized Levenshtein Distance to quantify similarities between string tokens. This measurement could also effectively minimize human errors such as typos.

Equality and Availability of Zip Code. This element may exist or not exist in a reference source. If unavailable, it does not mean it is not equal, means it is impossible to compare. Therefore, we consider three states: equal, unequal, unavailable.

Equality Tests for Base Number, Prefix and Postfix. For these address elements, the only condition for similarity between candidate and input is equality of their values, and no distance could be defined. Furthermore, data sources always contain these elements. Thus, we only considered equality test for each of them.

In addition, the following features are considered too: the reference type (TIGER, OA, Parcels and OSM), input zip code, and the response class. The response class originally had two values based on whether candidate is correct or wrong. By this type of classifying, as long as candidates are correct they are considered completely same by classifier. However, for minimizing the spatial error, we expand the response class definition to multiple classes with shorter ranges. This idea is very helpful for choosing the better candidate with less spatial error where several potentially correct candidates exist.

\section{Gradient Tree Boosting Based Classifier}

We use Gradient Tree Boosting based Classifier to train a prediction model. Gradient Boosting is a machine learning technique which generally uses weighted sum of multiple weak prediction models (decision trees) to predict on new datasets. Since relationships between parameters in this problem is nonlinear, decision trees as fundamental prediction models are a perfect fit.

Training. EaserGeocoder will provide a one-time training phase by learning relatively small decision trees as weak prediction models with according weights. This model solves the overfitting problem in learning mostly by using sub-samples of training set in each step. This feature is useful in problems that training sets and test sets have less similarity [25, 26]. After training with the ground truth dataset, the classifier learned about one hundred and fifty small decision trees. A sample decision tree is shown in Figure 4.

Classification of Input Address. After training, given an input address and the set of candidates generated from multiple references, a feature vector (same as training except response class) will be sent to the classifier, and a probability is assigned to each candidate based on underlying decision trees. The final result will be the candidate with the highest probability. The classification step will be the last step of the geocoding pipeline, Figure 2 .

\subsection{Scalable Geocoding}

The geocoding process takes one input address at a time and each input address forms a natural unit for parallelization. Parallel processing can be implemented in difference approaches. For single node based setup, we consider a master-slave approach; for a cluster based setup, we can take a MapReduce based approach.

Master Slave Based Parallelization In this approach, the database will be setup on a single node. A coordinator thread is responsible for creating, initializing and handling other threads. This thread reads input file with addresses to be geocoded, and creates one 
SIGSPATIAL'17, November 7-10, 2017, Los Angeles Area, CA, USA worker thread for each input address, initializes and starts the thread. When the worker thread finishes its work, the coordinator will process its output. The coordinator is also responsible for maximizing usage of active threads for boosting throughput of system. Each worker thread will perform all the steps discussed above and has direct access to the search engine. As all worker threads search the same database, disk I/O could be a potential bottleneck. Solr provides multiple caching capabilities to increase the efficiency, which can be easily customized. The estimated size of combined reference data sources is about 6GB for New York State, and about $100 \mathrm{~GB}$ for the nation. The size of indexes will need additional storage. Considering the decreasing cost of computer memory, modern computing nodes can easily provide sufficient memory to support caching. To maximize performance, we choose the total number of threads twice that of the number of CPUs.

MapReduce Based Parallelization Apache Solr includes the ability (Solr Cloud) to set up a cluster of Solr servers that combines fault tolerance and high availability by sharding and cluster scheduling. As our goal is high efficiency and scalability, we propose a Hadoop based approach for parallel geocoding for a cluster environment. Since the total size of data sources is not huge, instead of sharding, we can setup a Solr server on every node with all data sources loaded to avoid communication overhead, as a local query on a node only needs to search the local Solr database. The input collection of addresses to be geocoded will be pre-loaded into Hadoop Distributed File System. As each address forms a unit for parallelization, this approach will be embarrassingly parallel. The geocoding process can be implemented as a simple mapper only job, with a mapper to geocode a address, with the key as the record number, and value as address string. The output of the mapper will be the record number and geolocation. We will not discuss the implementation and experiments details on this approach due to space limit.

\section{EXPERIMENTS}

Finding a large enough ground truth for evaluation is a very challenging task. We take commercial results for comparison as as they usually acquired high quality databases. Besides, to avoid bias from a single vendor, we also created a fourth one combining the results (Google, Here, and MapQuest) named "Consolidation", which has higher agreement and could be closer to the ground truth. In this section, we compare the accuracy and positional errors of EaserGeocoder with well-known commercial and open source geocoders and the consolidated one from multiple commercial vendors (6.2). We then discuss pricing and limitations of commercial ones (6.3). Furthermore, we analyze contribution of different data sources (6.4). Finally, we measure scalability of our system in terms of input size and number of threads (6.5)

\subsection{Experimental Setup}

Testing Dataset. We collected 18,890 residential addresses by crawling one major real-estate web site for random house locations in New York State.

Hardware. We performed all experiments using a single cluster node with 22 physical cores $(2 \mathrm{x}$ Intel(R) Xeon(R) processor E5Ây2699 v3 at $2.20 \mathrm{GHz}$ ) and 88 virtual cores, 5TB hard drive at
S. Rashidian et al.

Table 1: Accuracy Comparison of Geocoders in Percentage

\begin{tabular}{|c|c|c|c|c|}
\hline Name/Source & Google & Here & MapQuest & Consolidation \\
\hline Google & - & 97.18 & 96.55 & 99.10 \\
\hline Here & 94.88 & - & 97.17 & 99.26 \\
\hline MapQuest & 94.52 & 97.16 & - & 98.82 \\
\hline $\begin{array}{c}\text { Easer- } \\
\text { Geocoder }\end{array}$ & $\mathbf{9 6 . 4 6}$ & $\mathbf{9 7 . 1 2}$ & $\mathbf{9 5 . 8 2}$ & $\mathbf{9 7 . 9 3}$ \\
\hline GeoServices & 94.68 & 95.30 & 94.71 & 96.43 \\
\hline Nominatim & 54.74 & 54.15 & 54.08 & 55.68 \\
\hline Geonames & 82.65 & 83.20 & 83.53 & 84.40 \\
\hline DST & 89.05 & 89.50 & 89.52 & 90.71 \\
\hline
\end{tabular}

Table 2: Spatial Error Comparison of Geocoders in Meters

\begin{tabular}{|c|c|c|c|c|}
\hline Name/Source & Google & Here & MapQuest & Consolidation \\
\hline Google & - & 24.29 & 55.52 & 18.37 \\
\hline Here & 24.29 & - & 55.20 & 17.67 \\
\hline MapQuest & 55.85 & 55.20 & - & 35.86 \\
\hline $\begin{array}{c}\text { Easer- } \\
\text { Geocoder }\end{array}$ & $\mathbf{3 1 . 0 8}$ & $\mathbf{2 6 . 8 5}$ & $\mathbf{5 3 . 3 0}$ & $\mathbf{2 6 . 9 0}$ \\
\hline GeoServices & 35.75 & 31.08 & 51.16 & 29.02 \\
\hline Nominatim & 65.55 & 63.88 & 57.98 & 58.09 \\
\hline Geonames & 93.38 & 91.78 & 75.32 & 83.58 \\
\hline DST & 87.42 & 85.47 & 71.36 & 77.39 \\
\hline
\end{tabular}

7200rpm and 128GB memory. Apache Solr 6.2.0. is used as our database server.

\subsection{Comparison with Other Geocoders}

We compare accuracy and spatial error of EaserGeocoder with major geocoders including commercial ones (Google, Here, MapQuest) and open source ones (GeoServices ${ }^{1}$, Nominatim, Geonames and $\mathrm{DST}^{2}$ ) [5-8, 10, 13, 24].

As ground truth geolocations are not available for large scale testing data, we choose three well known geocoders (Google, MapQuest and Here) alternatively as baseline for comparison. Furthermore, since none of them would necessarily produce the actual ground truth, we generate another consolidated baseline by aggregating results of all three systems based on following processing: 1) For each input address, if all three geocoding systems return similar points, we take the centroid of them as the final geolocation; 2) If the results are far from each other and there is no consensus, which may imply a potential problem of the address, we simply discard this address from the testing dataset; 3) Lastly, if two results are similar and the other one varies dramatically, we take the centroid of the first two as the geolocation. The similarity threshold between different results is set to 100 meters.

Geocoding accuracy is a common challenge for geocoding. In public health studies, geolocations represent patients' neighborhood and thus a reasonable deviation is still acceptable. Therefore, we define the accuracy as percentage of responses within 400 meters from the ground truth or baseline. Furthermore, as mentioned earlier, spatial error is defined as average of distances from all correct responses to their ground truth geolocations.

\footnotetext{
${ }^{1}$ Texas A\&M GeoServices

${ }^{2}$ Data Science Toolkit
} 
Effective Scalable and Integrative Geocoding

Table 3: Cost of Geocoding Based on Input Size

\begin{tabular}{|c|c|c|c|}
\hline Name & \multicolumn{3}{|c|}{ Price of transactions in \$ } \\
& $\mathbf{2 0 0 , 0 0 0}$ & $\mathbf{5 0 0 , 0 0 0}$ & $\mathbf{1 , 0 0 0 , 0 0 0}$ \\
\hline Google & 100 & 250 & 500 \\
\hline MapQuest & 399 & 799 & N/A \\
\hline Here & 119 & 499 & 449 \\
\hline GeoServices & $300-350$ & 700 & 1200 \\
\hline
\end{tabular}

Table 4: Comparison of Geocoding Results Using Different Data Sources

\begin{tabular}{|l|l|l|l|}
\hline Name & $\begin{array}{l}\text { Geolocation } \\
\text { level }\end{array}$ & $\begin{array}{l}\text { Accuracy } \\
\mathbf{\%}\end{array}$ & $\begin{array}{l}\text { Spatial } \\
\text { Error }(\mathbf{m})\end{array}$ \\
\hline TIGER & Street Level & 91.51 & 91.20 \\
\hline Tax Parcels & Rooftop & 71.93 & 35.08 \\
\hline OA & Rooftop & 65.96 & 32.01 \\
\hline SAM & Rooftop & 69.72 & 35.72 \\
\hline EaserGeocoder & Combined & $\mathbf{9 6 . 4 6}$ & $\mathbf{3 1 . 0 8}$ \\
\hline
\end{tabular}

Table 1 summarizes the measured accuracy of all geocoders with respect to different sources. Each column (2-4) represents the overall accuracy with one representative geocoder as the baseline (e.g., column 3 takes Here as baseline.) EaserGeocoder outperforms all open source geocoders and is comparable to well-known geocoding systems on geocoding accuracy. Table 2 shows comparison of spatial error. Again, EaserGeocoder outperforms all open source geocoders, and is comparable to commercial ones. Based on these two metrics, EaserGeocoder which relies on completely free data sources is in competition with big commercial geocoding systems.

\subsection{Cost and Limitations of Commercial Geocoders}

As discussed earlier, one of the problems with commercial geocoders is the high cost which could be overwhelming for researchers or small organizations to geocode massive address datasets.

Geocoding one million addresses could cost between 450-1600USD using a commercial system, and 100 million addresses could cost up to $160 \mathrm{~K}$ USD. Table 3 summarizes the cost of geocoding 1 million addresses with different geocoders.

Besides, most commercial systems put constraints on daily usage limit, which prevents high throughput geocoding. For instance, Google has a limit of 50 transactions per second and 100,000 transactions per day. Therefore, for one million addresses, geocoding process takes at least 10 days; and geocoding 100 million could take years. With a self-installed system like EaserGeocoder, we can have full control and achieve fast geocoding. As discussed later, it will take only 34 minutes for geocoding 1 million addressses, and less than 57 hours for geocoding 100 million addresses.

Finally, most geocoders provide Web only APIs to support geocoding through uploading data to their own servers. This creates a major privacy problem due to HIPAA rules.

\subsection{Comparison of Data Sources}

Each open data source has its pros and cons which will have impact on spatial accuracy and spatial error for geocoding. We perform a study to compare the results from different reference data sources.
SIGSPATIAL'17, November 7-10, 2017, Los Angeles Area, CA, USA

Based on results shown in Table 4, as predicted, among the four references, TIGER has the highest accuracy but comes with largest spatial error. On the other hand, exact-match references have lower accuracy but smaller error. The interesting point is that, OA, as a community contributed dataset, has the best spatial accuracy.

Through integrative geocoding, EaserGeocoder outperforms any single reference and achieves best accuracy (96.46\%) and smallest spatial error $(31.08 \mathrm{~m})$. Among the final results of EaserGeocoder, $40.40 \%$ comes from Tax Parcels, $35.83 \%$ comes from SAM, $12.21 \%$ comes from OpenAddresses, and $11.56 \%$ comes from TIGER. Although TIGER could produce more than $90 \%$ of the results, EaserGeocoder picks only $12 \%$ from it and selects most results from exact-match references, which minimizes spatial error.

The histogram in Figure 5a, shows distribution of distances from the ground truth for this test dataset. Most results have less than $100 \mathrm{~m}$ distance to their true geolocation.

\subsection{Scalability of EaserGeocoder}

Scalability is among the most desired features of geocoding systems. We perform two experiments to study the scalability of EaserGeocoder based on master-slave based implementation. First, we use the same computing resource by increasing the number of queries (addresses to be geocoded) and measure the running time. As shown in Figure 5b, the timing increases linearly. Second, for a fixed number of queries $(100,000)$, we increase the number of threads. Figure $5 \mathrm{c}$ shows the running time continuously drops with increasing number of threads, and the curve can be fit into a close power function which shows high scalability.

Based on the performance, geocoding 100 million addresses will take about 3378 minutes. To geocode addresses of 200 million patient addresses for New York SPARCS data, it will take 113 hours. Note that this is the performance based on a single node. Based on a MapReduce based approach (Section 5.5), we expect similar scalability using a cluster of computing nodes, which can achieve high throughput (future work).

\section{ACKNOWLEDGEMENTS}

This research is supported in part by grants from National Science Foundation ACI 1443054 and IIS 1350885.

\section{CONCLUSION}

Spatial big data based public health studies using individual patient addresses pose a unique challenge for geocoding research due to the requirement of privacy protection and massive number of addresses. In this paper, we show that multiple open address data sources provide rich address data complementing each other, and provide a unique opportunity for developing a cost-effective and open source geocoding solution for massive address datasets. We propose an integrative geocoding approach EaserGeocoder to take advantage of the characteristics of each reference data source to generate result candidates for an input address. Instead of traditional fixed weighting based approach, we propose a machine learning based approach to automatically choose the best answer among all the candidates by learning from public addresses using a comprehensive set of address features. Our approach is highly scalable with parallel processing. Our extensive experiments demonstrate 


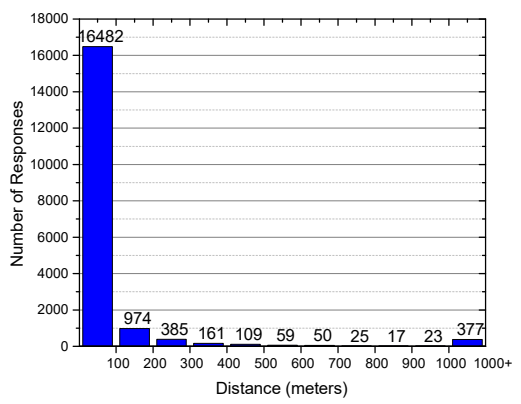

(a) Histogram of Responses

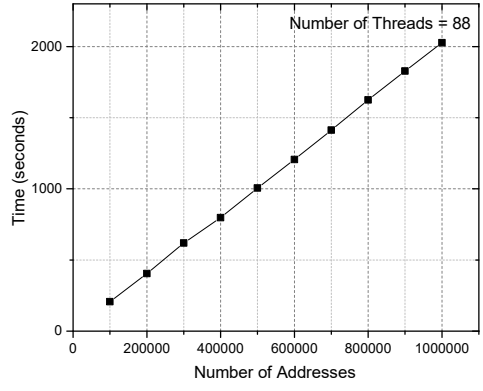

(b) Scalability: Time vs Input Size

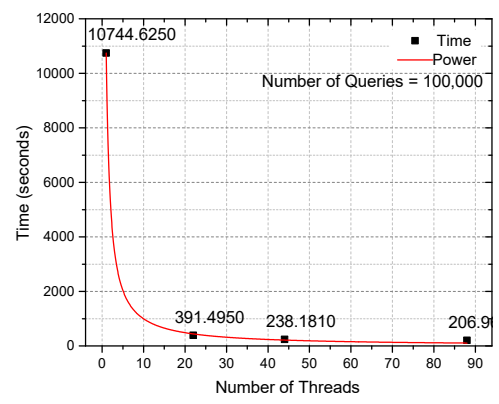

(c) Scalability: Time vs Threads that EaserGeocoder outperforms other open source systems and is comparable to commercial ones. EaserGeocoder will be open source for the research communities.

We observe that driving by open data initiatives by the governments and communities' enthusiasm, there are increasing effort on producing public address data sources with continuously increased amounts of objects and improved accuracy. We envision that our integrative geocoding system will benefit from the trend to achieve higher quality geocoding in the future.

\section{REFERENCES}

[1] 2009. WhyUseSolr - Solr Wiki. https://wiki.apache.org/solr/. (09 2009).

[2] 2016. Physician and Other Supplier Data CY 2013. https://www.cms.gov/ Research-Statistics-Data-and-Systems/Statistics-Trends-and-Reports/MedicareProvider-Charge-Data/Physician-and-Other-Supplier2013.html. (Dec. 2016).

[3] 2016. SPARCS. https://www.health.ny.gov/statistics/sparcs/. (2016).

[4] 2016. What is Geocoding? http://desktop.arcgis.com/en/arcmap/10.3/guidebooks/geocoding/. (2016)

[5] 2017. Data Science Toolkit. http://www.datasciencetoolkit.org/. (2017).

[6] 2017. GeoNames. http://www.geonames.org/. (2017).

[7] 2017. Google Maps Geocoding API. https://developers.google.com/. (2017)

[8] 2017. HERE Geocoder API. https://developer.here.com/. (2017).

[9] 2017. HIPAA PHI: List of 18 Identifiers and Definition of PHI http://cphs.berkeley.edu/hipaa/hipaa18.html. (2017).

[10] 2017. MapQuest Developer Network. https://developer.mapquest.com/. (2017).

[11] 2017. NYS GIS Clearinghouse - NYS Address Points. (April 2017). Retrieved May 17,2017 from http://gis.ny.gov/gisdata/inventories/details.cfm?DSID=921

[12] 2017. OpenAddresses - Download Data. http://results.openaddresses.io/. (2017)

[13] 2017. OpenStreetMap Nominatim. http://nominatim.openstreetmap.org/. (2017).

[14] 2017. OpenStreetMap Wiki. http://wiki.openstreetmap.org. (2017).

[15] 2017. TIGER Products - Geography - U.S. Census Bureau. (April 2017). Retrieved May 26,2017 from https:/www.census.gov/geo/maps-data/data/tiger.html

[16] Rahul Bakshi, Craig A Knoblock, and Snehal Thakkar. 2004. Exploiting online sources to accurately geocode addresses. In Proceedings of the 12th annual ACM international workshop on Geographic information systems. ACM, 194-203.

[17] Pavel Berkhin, Michael R Evans, Florin Teodorescu, Wei Wu, and Dragomir Yankov. 2015. A new approach to geocoding: BingGC. In Proceedings of the $23 \mathrm{rd}$ SIGSPATIAL International Conference. ACM, 7.

[18] Michael R Cayo and Thomas O Talbot. 2003. Positional error in automated geocoding of residential addresses. International journal of health geographics 2 , 1 (2003), 10.

[19] Abhranil Chatterjee, Janit Anjaria, Sourav Roy, Arnab Ganguli, and Krishanu Seal. 2016. SAGEL: smart address geocoding engine for supply-chain logistics. In Proceedings of the 24th ACM SIGSPATIAL International Conference. ACM, 42.

[20] Xin Chen, Hoang Vo, Ablimit Aji, and Fusheng Wang. 2014. High performance integrated spatial big data analytics. In Proceedings of the 3rd ACM SIGSPATIAL International Workshop. ACM, 11-14.

[21] Xin Chen and Fusheng Wang. 2016. Integrative Spatial Data Analytics for Public Health Studies of New York State. In AMIA Annual Symposium Proceedings, Vol. 2016. American Medical Informatics Association, 391.

[22] New York State Geographic Information Systems Clearinghouse. 2014. NYS GIS Clearinghouse - NYS Tax Parcels. (Nov. 2014). Retrieved Feb 20,2017 from
http://gis.ny.gov/gisdata/inventories/details.cfm?DSID $=1300$

[23] Steve M Dearwent, Robert R Jacobs, and John B Halbert. 2001. Locational uncertainty in georeferencing public health datasets. Fournal of Exposure Science and Environmental Epidemiology 11, 4 (2001), 329.

[24] Goldberg DW. 2017. Texas A\&M University Geoservices. http://geoservices.tamu.edu. (2017).

[25] Jerome H Friedman. 2001. Greedy function approximation: a gradient boosting machine. Annals of statistics (2001), 1189-1232.

[26] Jerome H Friedman. 2002. Stochastic gradient boosting. Computational Statistics \& Data Analysis 38, 4 (2002), 367-378.

[27] DW Goldberg, JN Swift, and JP Wilson. 2008. Geocoding best practices: Analysis of Geocoding User Requirements. Los Angeles, CA: University of Southern California GIS Research Laboratory (2008).

[28] Daniel W Goldberg. 2008. A geocoding best practices guide. (2008).

[29] Daniel W Goldberg. 2011. Improving Geocoding Match Rates with SpatiallyVarying Block Metrics. Transactions in GIS 15, 6 (2011), 829-850.

[30] Daniel W Goldberg and Myles G Cockburn. 2010. Improving geocode accuracy with candidate selection criteria. Transactions in GIS 14, s1 (2010), 149-176.

[31] Daniel W Goldberg, John P Wilson, and Craig A Knoblock. 2007. From text to geographic coordinates: The current state of geocoding. URISA-WASHINGTON DC- 19, 1 (2007), 33.

[32] Geoffrey M Jacquez. 2012. A research agenda: does geocoding positional error matter in health GIS studies? Spatial and spatio-temporal epidemiology 3, 1 (2012), $7-16$.

[33] Ludovic Moncla, Walter Renteria-Agualimpia, Javier Nogueras-Iso, and Mauro Gaio. 2014. Geocoding for texts with fine-grain toponyms: an experiment on a geoparsed hiking descriptions corpus. In Proceedings of the 22nd ACM SIGSPATIAL International Conference. ACM, 183-192.

[34] U.S. Department of Health \& Human Services. 2015. Health Information Privacy | HHS.gov. https://www.hhs.gov/hipaa/index.html. (2015).

[35] Gerard Rushton, Marc P Armstrong, Josephine Gittler, Barry R Greene, Claire E Pavlik, Michele M West, and Dale L Zimmerman. 2006. Geocoding in cancer research: a review. American journal of preventive medicine 30, 2 (2006), S16-S24.

[36] Xuan Shi, Bowei Xue, and Imam M Xierali. 2016. Identifying the Uncertainty in Physician Practice Location through Spatial Analytics and Text Mining. International Journal of Environmental Research and Public Health 13, 9 (2016), 930 .

[37] Grant Ian Thrall. 1999. The future of GIS in public health management and practice. Journal of Public Health Management and Practice 5, 4 (1999), 82.

[38] Eric A Whitsel, P Miguel Quibrera, Richard L Smith, Diane J Catellier, Duanping Liao, Amanda C Henley, and Gerardo Heiss. 2006. Accuracy of commercial geocoding: assessment and implications. Epidemiologic Perspectives \& Innovations 3, 1 (2006), 8 .

[39] Duck-Hye Yang, Lucy Mackey Bilaver, Oscar Hayes, and Robert Goerge. 2004. Improving geocoding practices: evaluation of geocoding tools. fournal of medical systems 28, 4 (2004), 361-370.

[40] PA Zandbergen, TC Hart, KE Lenzer, and ME Camponovo. 2012. Error propagation models to examine the effects of geocoding quality on spatial analysis of individual-level datasets. Spatial and spatio-temporal epidemiology 3, 1 (2012), 69-82.

[41] Paul A Zandbergen. 2008. A comparison of address point, parcel and street geocoding techniques. Computers, Environment and Urban Systems 32, 3 (2008), 214-232.

[42] Paul A Zandbergen. 2009. Geocoding quality and implications for spatial analysis. Geography Compass 3, 2 (2009), 647-680. 